\title{
A Model of Global Cooperation to Enable Intelligent Metrology Applications by Means of an Integrated Teleoperation System
}

\author{
http://dx.doi.org/10.3991/ijoe.v11i3.4545 \\ N.M. Durakbasa ${ }^{1}$, J.M. Bauer ${ }^{1,2}$, G. Bas ${ }^{1}$ and D. Riep ${ }^{1}$ \\ ${ }^{1}$ Vienna University of Technology (TU-Vienna), Vienna, Austria \\ ${ }^{2}$ National Technological University (UTN-FRBA), Buenos Aires, Argentina
}

\begin{abstract}
Today's advanced production industry faces challenges of increasing quality and competition requirements in the global market. The new aspects of cooperation between the production industry and metrology laboratories enable a development capacity providing an information highway and expertise exchange by accommodating rising demand of services. This paper proposes a novel approach to develop a global network on two different continents that will moreover be a model for the industry without any limits of location, time difference or facility.
\end{abstract}

Due to the high cost of special infrastructure, equipment and expertise for production of complex components, this study also proposes a strategic approach to develop a remotely controlled high precision metrology system with high accuracy as well as with the smallest measurement uncertainties for the micro- and nanotechnologies and intelligent integrated management system applicable in advanced manufacturing industry. This will provide a roadmap for the industrial applications as well as in educational organizations with the developed and integrated low-cost telepresence and teleoperation system.

Index Terms-Advanced production industry, high precision metrology, teleoperation, telepresence.

\section{ThE CURRENT CONDITION}

The advanced manufacturing industry has progressed to innovative products and industrial applications with a broad range of areas by means of developments in micro and nanotechnology. During the last few decades, micro and nanotechnology has changed from a technology only applied in research laboratories to a technology that is practiced in manufacturing and industrial applications.

The European Commission, which has defined nanotechnology as one of the Key Enabling Technologies for growth and jobs, has supported nanotechnology projects over the past decade by the Seventh Framework Programme (FP7) with the largest single share of funding for nanotechnology, of EUR 896 millions for the period 2007-2011 and now continues by the new founding initiative Horizon2020 [1, 2, 3].

However, the innovative products with structures in the micro- and nano-scale represent interdisciplinary set of complications in integration processes with quality problems. As the challenges of improving quality of consumer goods and further advanced products are inevitable part of the next-generation manufacturing, convenient solutions are required to be developed that will enable to overcome the challenges. These "grand challenges" have been recognized and are addressed by the Europe 2020 strategy [4].

The new aspects of cooperation between the research institutions and the manufacturing industry will provide a development capacity for high quality and innovative products. The development of information highway, technology and advanced engineering data exchange techniques make global information systems by means of collaborative and interactive environment [5]. Hence, this paper proposes a model of remotely controlled research facility for educational purposes that will moreover serve as a system applicable in the advanced manufacturing industry.

The rapid development of internet technology has enabled additional approaches in laboratory and educational systems by applying telepresence [6]. Effectiveness of teleoperation and telepresence can be maintained by development of necessary expertise on the role and value of the laboratory work [7].

Moreover, universities have to reconsider their position as organizations that provide exclusive education to evolve by integrate and exploit advanced and flexible ways of their relevant capabilities, by means of their units, laboratories with both real and virtual technologies [8].

\section{A Model Of Global CoOperation}

\section{A. Advanced Production Industry}

Cooperation of the next-generation manufacturers with technology developers has made an indispensable requirement for precision in micro- and nano-scale in the production line. The micro-components and nanostructures have opened now a new era in the geometrical product specification standards that covers not only the quality aspects but also mandatory efficient integration of sub-components to create the product. The needs of the next-generation manufacturing industry for ultra-high precision engineering and workpieces with a surface roughness less than few nanometers call for measurement instrumentation that can be applied reliably in modern production processes, together with international standards defining parameters and tolerances in the nanometer scale, using in a high accurate environment such as high precision metrology laboratory. 
The production of very precise components goes hand in hand with the development of the necessary metrology, and a wide range of measuring instruments has been devised to cater for the evaluation of surfaces and structures down to the $0.1 \mathrm{~nm}$ level. Particularly noteworthy are the stylus profilometer, the atomic force microscope, the scanning tunneling microscope, the polarizing interferometer, the laser profilometer and the X-ray interferometer. This powerful array of instruments provides a measuring capability which ranges from $50 \mathrm{pm}$ to $15 \mathrm{~mm}$ in surface amplitude and from $50 \mathrm{~nm}$ to $250 \mathrm{~mm}$ in surface wavelength, and techniques for roundness measurements to $1 \mathrm{~nm}$ and displacement calibration to 10 $\mathrm{pm}$, traceable to the national standards of length [9].

The concept of remote controlled high precision laboratory may help industry for the robust and repeatable nanofabrication of structures with atomic control of size, geometry, shape, spatial position, location, orientation, run out and chemical composition.

The increased international networking of research will not only support the transfer of knowledge, but also utilise potential synergies in the field of laboratory infrastructure in partner institutions. The ongoing rapid development of information technology and global networks as well as the convergence of virtual and real worlds are the key factors in the modern industrial production. Production processes will be designed to dynamically and efficiently. The production of the future will be characterized by a fully integrated and highly flexible production chain with integrated intelligent systems under guidance of international standards [10].

The quality management system that can be integrated with the environmental and energy management systems in compliant with the international standards is the fundamental strategy to obtain the required operation conditions for a competitive manufacturing organization (Figure 1) [11, 12, 13].

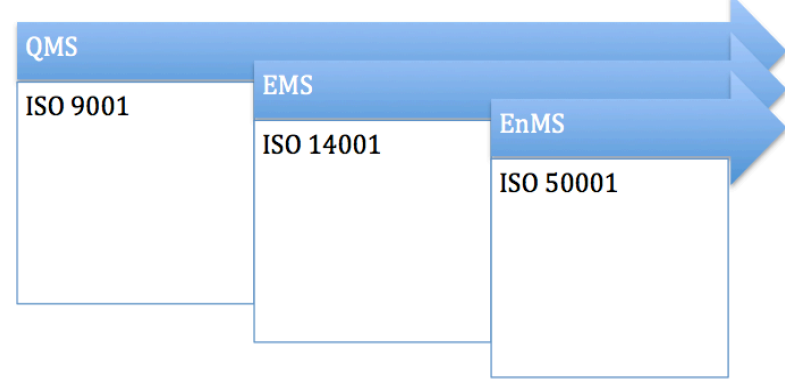

Figure 1. The integrated management system of quality, environment and energy

The management system is modeled based on the continuous improvement approach. The closed loop model identifies the Plan-Do-Check-Act processes to be determined, audited, documented and improved as the strategy develops towards advanced manufacturing technology such as intelligent management system integration.

The approach of overcoming the challenges that can occur is proposed in this work by means of process management toolbox analysis. The management system to be implemented in a manufacturing plant is modeled and represented in the Figure 2.

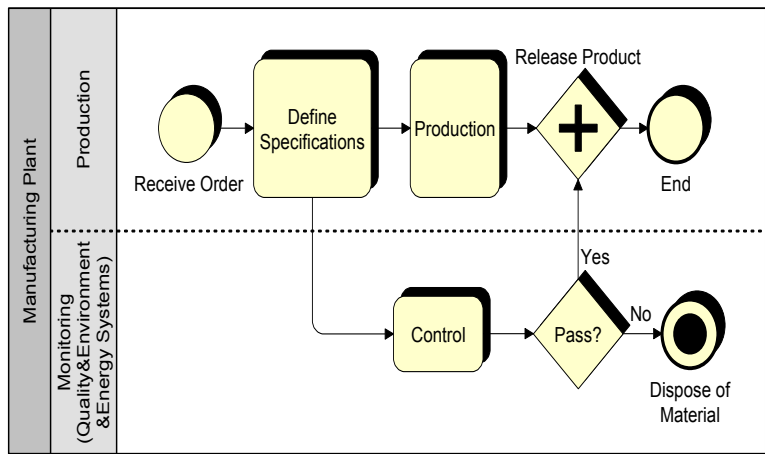

Figure 2. The process flow of integrated management approach in a manufacturing plant

The process flow of integrated management approach in a manufacturing plant offers the case studies and future estimations to be processed before. The process management simulation toolbox [14] provides the reporting system that is used for calculation of development aspects in the plants in terms of cost, labor use and resources, which is applicable to any kind and size of organizations. The approach of modeling enhances the efficiency of modern technology integration plans in the industrial plants.

The next-generation manufacturing industry integrating the teleoperation with intelligent automation requires quality control for their operation. Metrology as the measurement science provides the functional methodology for quality control under the defined specifications and standards.

The quality assurance process starts with the data collection and evaluation using the measurement science methodology. When considering the teleoperation quality assurance, it is required to deal with complex, variable and dynamic control problems of the production process. Hence, the design system and other manufacturing processes must be considered as a whole while implementing the self-optimizing process. This approach can be summarized for an intelligent measurement process with the following tasks:

- Automatic intelligent measurement by using CNC metrology

- On-line and Off-line CNC programming of measuring instruments

- Automatic changing of workpieces

- Automatic changing of probes and sensors

- Automated evaluation of measuring results

- Sophisticated network system

The proposed solution methodology can be considered as a further step with a target of intelligent and economical manufacturing environment using the quality assurance cycle as represented in the Figure 3.

The operating models to the manufacturing plants are developing to keep up with the robotic and automation applications for advanced industrial processes [15]. The simulation of the control systems for development of computer aided, automated production are demand driven aspects that states the future of the manufacturing industry [16]. 
PAPER

A Model of Global Cooperation to Enable Intelligent Metrology Applications by MeAns of An...

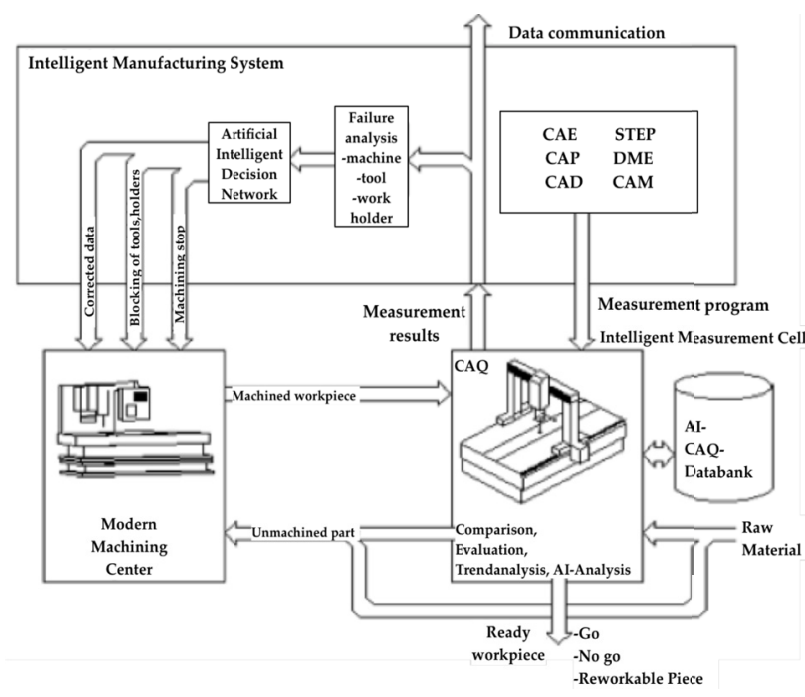

Figure 3. The intelligent manufacturing system

\section{B. The Requirements of High Precision Measurement}

Advanced technologies in precision engineering, machining, biotechnology, optics, electronics, materials, will increasingly require high-accuracy qualification of mechanical and electrical properties in addition to physical dimensions. The need for new high precision and innovative measurement techniques are indispensible to provide reliable measurement results with small uncertainties at the micro and nano-scale production concurrent with the latest nanotechnology developments.

Industry may require high-quality nanometrology rooms for the robust and repeatable nanofabrication of structures with atomic control of size, shape, spatial position, and chemical composition.

It is very significant and essential to make scientific research in accordance with global standards, so that the measurement results are to be acknowledged internationally in the scientific world. In addition to this, global standards help the accuracy on sub-micrometer and nanometer scales to improve. This creates the need of a measurement laboratory, which is carefully and properly designed and ensures high accuracy for measuring with the smallest measurement uncertainties. Making certain of consistent and reproducible environmental condition is the key factor for designing and building such a laboratory.

The most important environmental measurement influences are:

- temperature (thermal conduction, convection and radiation)

- vibrations

- humidity

- pollution

The structural organization of a precision measurement room ensures that most of these disturbing influences will be reduced and kept constant. The High precision measurement room - Nanometrology Laboratory of the Vienna University of Technology demonstrates the demands for such a building and features of technical realization (Figure 4).

The aim for planning a high precision measurement room is to minimize by structural organization most of the disturbing influences on the measuring instruments installed in the measurement room and the measuring process [17].

The High Precision Measurement Room Nanometrology Laboratory located in the basement of the main building of the Vienna University of Technology, is of the surrounding buildings separated mechanical and structural -concept of "room-in-room"- and has solid walls and a concrete slab (30 cm thick), which is passive vibration isolated from the environment and in such a manner it also prevents a transfer of building oscillations through the base plate on the installed measurement devices.

The system consists of "High precision measurement room - Nanometrology Laboratory", control room, sluice, entrance hall and machine room.

To obtain reliable measurement results, an environment with defined values for temperature, humidity, air pressure, air speed and particles in the laboratory is necessary. Through a powerful air conditioning system, there will be the continuous monitoring and control to perform the constant environmental conditions in the laboratory. There exists a moderate overpressure in the laboratory. This slightly overpressure of about $10 \mathrm{~Pa}$ in the measurement Laboratory is to prevent the irruption of unfiltered and unconditioned air when doors are opened.

Only an appropriately designed laboratory with consistent and reproducible environmental conditions may guarantee precise measurements with high accuracy and the smallest measurement uncertainties. Hence, this study presents a system of high precision measurement facility by taking advantage of the potential to optimize the use of own technological resources at an university department. It is of course an interdisciplinary challenge to implement a working platform creatively based on these concepts.

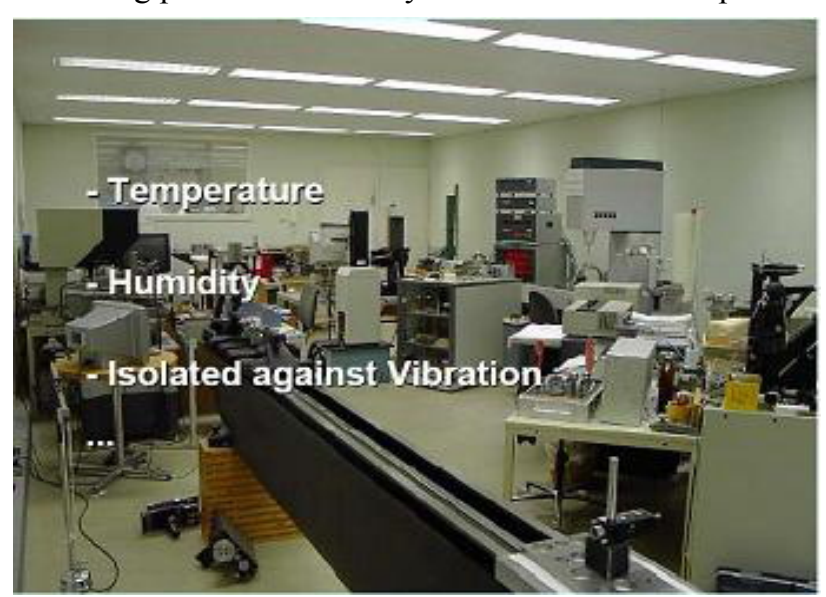

Figure 4. High precision measurement room - Nanometrology Laboratory of Vienna University of Technology

\section{The Model Of A Global COOPERATION For High Precision METROLOGY ApPLiCATIONS}

In the field of precision metrology, measurement results are influenced by temperature, mechanical vibration, dust, pollution and humidity. The High Precision Measurement Room and Nanotechnology Laboratory at the Vienna University of Technology fulfils all the necessary requirements.

The teleoperation in this work gives us the possibility to operate sophisticated devices for particular workpieces. As the internet highway is more and more improved, the 
teleoperation and its usage develop rapidly. This empowers new opportunities in relevant fields and reduces the costs.

The telepresence widen the teleoperation to a distant environment and allows users to interact in a dynamic two-way method with a set of technologies in order to allow presence, operation of equipment and interaction with peers in both visual and audio format.

In order to reach the goal of remote laboratory concept, the first step is to build up a telepresence system in the high precision and nanotechnology laboratory, so that the remote experimental work is not only conducted in the framework of the measuring instrument software but also is supported by video and audio integration to the instrument room (Figure 5).

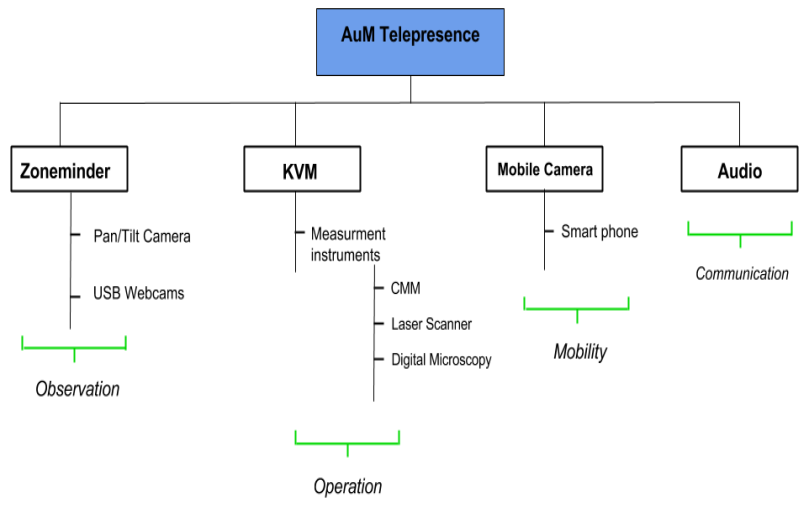

Figure 5. Main components of the telepresence system

There are four different main components of the telepresence system. Each of these four components is a significant part of approaches, which make alltogether an efficient telepresence system. These approaches are as following;

- observation

- operation

- mobility

- audio

In the high precision laboratory, there are various measuring instruments available. The telepresence system gives an overview to the laboratory activities so that the user is aware, if anybody is in the laboratory and/or working on any of the measurement instrument (Figure 6).

USB webcams, mobile cameras and pan-tilt (ip) cameras carry out monitoring of the laboratory and experiments. These cameras are configured and operated by an operator-pc, which was programmed as a zoneminder (zm).

Zoneminder is an integrated set of applications, which provide a complete surveillance solution allowing capture, analysis, recording and monitoring of any cetv or security cameras attached to a linux based machine. It is designed to run on distributions, which support the video for linux (v4l) interface and has been tested with video cameras attached to bttv cards, various usb cameras and also supports most ip network cameras.

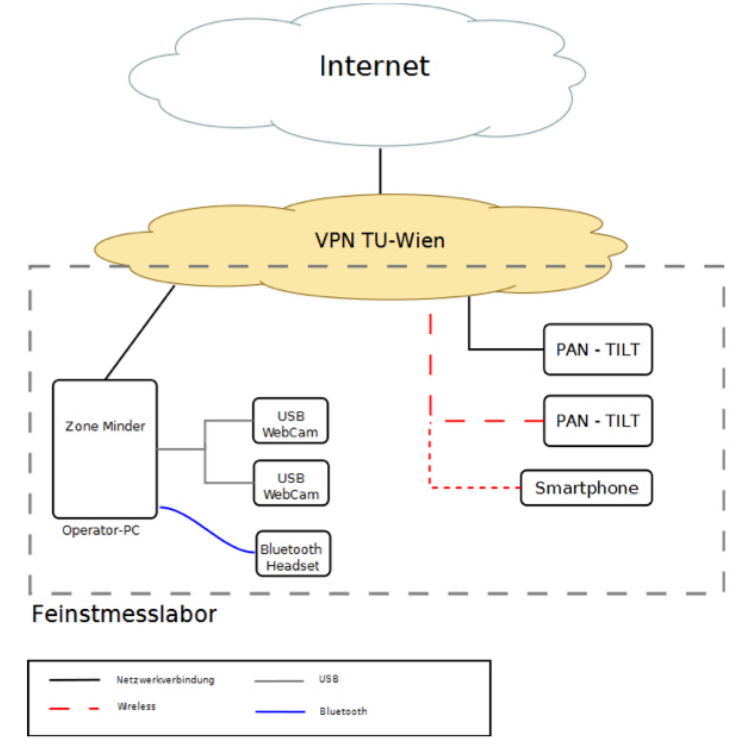

Figure 6. The components of observation system

\section{The Intelligent Metrology APPliCATIONS By TELEPRESENCE AND TELEOPERATION}

The time, cost and expertise for establishing metrology and mechanical laboratories is very high for many small and medium size enterprises. The facility infrastructure and the personnel expertise will be available in the global market as one of the targets in this development project.

The current status of the project focuses on automatic control of the high precision measurement instruments through the network established using a server. The operation requirements of remote control results an increase of the workload of the channel in the network. Hence, the bandwidth limitations generate a reduction speed of the process. To resolve this difficulty; a client server computing technology is used for the control of all the equipment in the laboratory. Establishing a paradigm in the Linux server enabled us to connect to the equipments, which is required for further applications.

The system established is based on the assumption that it works under the condition that each single operation is controlled and reported. The model of the working environment is a sample for any manufacturing plant that is prepared to integrate intelligent manufacturing machines in its environment (Figure 7). Cooperative activities in the laboratories create systematic knowledge transfer and data acquisition. Each process is carried out step by step in harmony with the required standards.

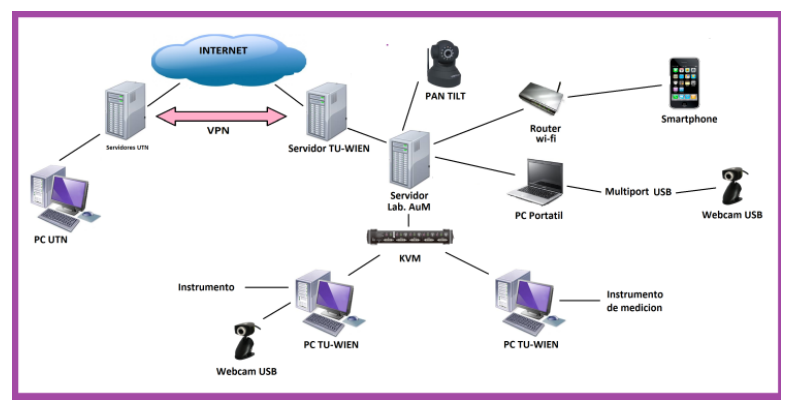

Figure 7. The overview of working environment 
PAPER

A Model of Global Cooperation to Enable Intelligent Metrology Applications by MeAns of An...

Implementation of the intelligent metrology cycle as the target, research and development process provides a model to establish at the universities and institutes. The experience obtained by integration of the process concept will create a roadmap for future developments.

The practical approach of this inter-university network project offers collaboration to any industrial or university organization without boundaries. As a part this strategy, the High precision measurement room - Nanometrology Laboratory is chosen as a node to create an interuniversity network. The developed platform serve not only as a practical experience of an intelligent metrology process model but also as an educational concept for the current students taking part in high precision measurement activities. Learning and continuous improvement are the basics of each module of the system. Once the targets are achieved, it is possible to implement the modules in another node using the systematic knowledge obtained throughout the project. Moreover, the cooperation between the local laboratories in Argentina enables the practical applications to develop and create a resource for future connection nodes that is already in the near future plans.

\section{A. Teleoperation with the 3D Laser Scanner}

$3 \mathrm{D}$ surface-scanning refers to the seizure of a workpiece's shape, or more formal the acquisition of a set of 3D coordinates on the workpiece's surface. In recent years, 3D surface scanning has become very important and its applications spread to various fields, like prototyping, in-process inspection, reverse engineering, medicine and so on.

The laser scanner (Figure 8) in the high precision laboratory for the applications is a specifically designed instrument for dental/medical applications. Its software, for that reason, has specifications for a 3D scanning of anatomical implants and digitizing of surfaces has a particular algorithm for these objects.

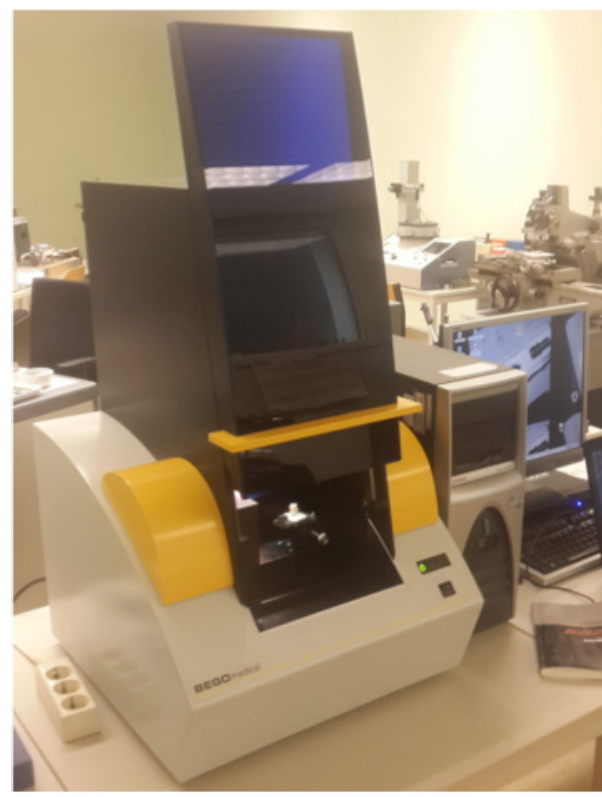

Figure 8. The 3D Laser Scanner

In this case, a tooth sample will be measured in the scanner. The workpiece is fixed in the center of the scanner and the uv filtered door is closed in order to prevent any hazardous light exposure to the experimenters. Once the workpiece and the machine are ready, rest of the measuring process is performed by teleoperation via the interface of the software implemented in the measuring device (Figure 9).

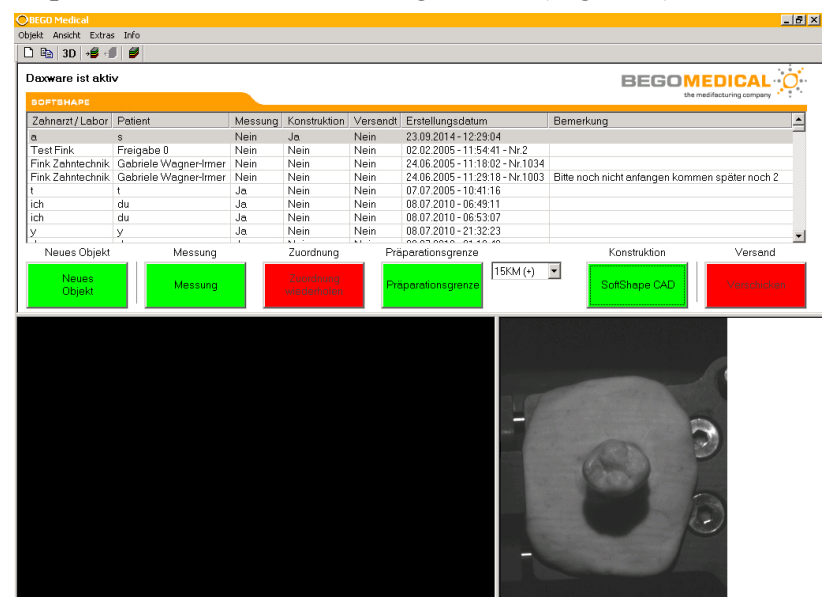

Figure 9. The screen shot of the remote controlled laser scanner measurement

After entering the relevant data, the device runs the scanning on the object and the software renders the acquired data from laser scan and gives the 3D CAD image of the object. After the 3D CAD image is obtained, modifications can be performed on the image of the tooth sample on another window of the dental-specialized software (Figure 10).
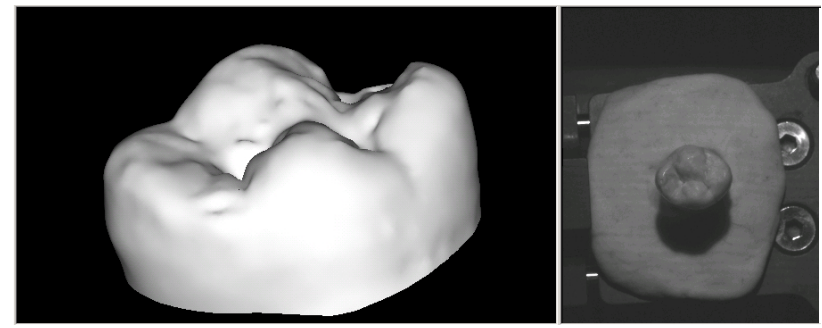

Figure 10. The 3D model of the tooth sample

\section{CONCLUSION AND Future WORK}

In this study, the High precision measurement room Nanometrology Laboratory at the Vienna University of Technology is chosen as a node to create an interuniversity network. The goal is not only to implement and observe real-time the tasks performed in the Laboratory but also to enable a model for industrial applications by means of active participation with telepresence in the measurement process with dynamic queries and actions.

The current status of the project focuses on moreover high precise measurement and data collection from other high precise measurement equipments such as the coordinate measurement machine. The practical approach of this inter-university network project offers collaboration to any industrial or university organization without boundaries.

Learning and continuous improvement are the basics of each module of the system. Once the targets are achieved, it is possible to implement the modules in another node using the systematic knowledge obtained throughout the project. The modern manufacturing industry integrating the intelligent automation solutions requires quality 
control for their operation. Metrology as the measurement science provides the functional methodology for quality control under the defined specifications and standards in this Laboratory environment.

Hence, the need of high precise measurement tasks and essential measurement know-how within the sophisticated production systems for manufacturing companies will be met with the proposed applications.

Besides, this study proposes a strategic approach to develop a smart integrated system applicable in manufacturing industry using the intelligent networking for the digital factory by firstly modeling, generating and experimenting an inter-university network that accesses, cooperates and operates at distance in the laboratory of distant research laboratories that can be applicable to all other industrial organizations. Hence, this project proposes a model of international collaboration based on the laboratory facility and offers also the industry a roadmap for possible technology applications.

The intelligent metrology applications will be the future solution for advanced manufacturing industry. As a result, this collaborative study with the target of practical approach to the measurement applications offer an innovative main aspect of the technology progress.

\section{REFERENCES}

[1] European Commission of the European Communities, Communication from the Commission to the European Parliament, the Council, the European Economic and Social Committee and the Committee of the Regions - "Preparing for our future: Developing a common strategy for key enabling technologies in the EU" $\{\operatorname{SEC}(2009) 1257\}, \operatorname{COM}(2009) 512$ final, Brussels, 30.09.2009.

[2] European Commission, Nanotechnology: the invisible giant tackling Europe's future challenges, Brussels, 2013.

[3] European Commission, Horizon 2020 Work Programme 2014 - 2015, European Commission Decision C (2014)4995, 22.July.2014.

[4] European Commission, The Grand Challenge, The design and societal impact of Horizon 2020, Brussels, 2013.

[5] P.H. Osanna, N.M. Durakbasa, J. M. Bauer, L. Krauter, Global International Cooperation of Collaborating Small and Medium Sized Enterprises to Achieve Total Quality Management, Proceedings Fourth International Working Conference, Belgrad, 27- 30.05.2007.

[6] C. Krause, U. Strunz, Vision system for teleoperating mobile robots, Telepräsenz bei mobilen Robotern, Forschung im

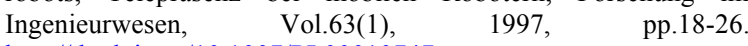
http://dx.doi.org/10.1007/PL00010747

[7] J. Ma, J. V. Nickerson, Hands-On, Simulated, and Remote Laboratories: A Comparative Literature Review, ACM Computing Surveys, Vol. 38, No. 3, Article 7, September 2006.
[8] S. Popescu, S. Brad, D. Popescu, The engineering study program as a customised product: barriers and directions for intervention, The 1st CIRP-International Seminar on Assembly Systems, Stuttgart, 15-17.November.2006.

[9] M.N. Durakbasa, P.H. Osanna, Development and State of the Art of Production Metrology for the 21st Century - From Micrometrology to Nanometrology and Picometrology. Modern Technologies in Manufacturing, Proceedings of the 6th International Conference MTeM, ISBN: 973-656-490-8, ClujNapoca, 2003.

[10] M.N. Durakbasa, G. Bas, Z. Özkan, A New Strategy for Energy A Proposal of an Energy Management Implementation Model for Productivity Enhancement within the Integrated Management Systems, Journal of the Institute of Management Services, 1 (2011), Volume 55 Number 2; pp. 28 - 30.

[11] ISO 9001:2008: Quality management systems - Requirements.

[12] ISO 14001:2004 + Cor. 1:2009: Environmental management systems - Requirements with guidance for use.

[13] ISO 50001:2011: Energy management systems - Requirements with guidance for use.

[14] iGrafx Inc., "iGrafx Process Modeling and Analysis 2013", 2013.

[15] P. Kopacek, Intelligent Manufacturing: Present State and Future Trends, Journal of Intelligent and Robotic Systems, 1999, Volume 26, pp. 217-229. http://dx.doi.org/10.1023/A:1008168605803

[16] M.N. Durakbasa, Geometrical product specifications and verification for the analytical description of technical and nontechnical structures, Department of Interchangeable Manufacturing and Industrial Metrology, TU-Vienna Austria, 2014.

[17] A. Weckenmann, W. Scharf, Conception of a high precision measurement room. XVI IMEKO World Congress, Vienna, 25.28. 09.2000, Proceedings, Vol. 8, pp. 315-319.

\section{AUTHORS}

N. M. Durakbasa is with the Vienna University of Technology (TU-Vienna), Vienna, Austria (e-mail: numan.durakbasa@tuwien.ac.at).

J. M. Bauer, is with the Vienna University of Technology (TU-Vienna), Vienna, Austria and National Technological University (UTN-FRBA), Buenos Aires, Argentina, (e-mail: jbauer@doc.frba.utn.edu.ar).

G. Bas is with the Vienna University of Technology (TU-Vienna), Vienna, Austria (e-mail: goekcen.bas@tuwien.ac.at).

D. Riepl is with the Vienna University of Technology (TU-Vienna), Vienna, Austria (e-mail: aum@ift.tuwien.ac.at).

This article is an extended and modified version of a paper presented at the International Conference on Remote Engineering \& Virtual Instrumentation (REV2015), held in Bangkok, Thailand, 25 - 28 February 2015.Submitted 20 March 2015.Published as resubmitted by the authors 04 May 2015. 